\title{
ARTICLE
}

-Cite this:

Received 00th January 2012, Accepted 00th January 2012

DOI:

\section{Cryogenic magneto-caloric effect and magneto- structural correlations in carboxylate-bridged Gd(III) compounds}

\author{
O. Roubeau, ${ }^{\mathrm{a},}{ }^{*}$ G. Lorusso, ${ }^{\mathrm{a}}$ S. J. Teat ${ }^{\mathrm{b}}$ and M. Evangelistia
}

Two new infinite coordination chain compounds $\left[\mathrm{Gd}\left(\mathrm{CH}_{3} \mathrm{CO}_{2}\right)_{3}(\mathrm{dmf})\right]_{\infty} \quad$ (1) and $\left.\left\{\left[\mathrm{Gd}\left(\mathrm{HO}\left(\mathrm{CH}_{2}\right)_{3} \mathrm{CO}_{2}\right)\right)_{3}\left(\mathrm{H}_{2} \mathrm{O}\right)\right] \cdot \mathrm{H}_{2} \mathrm{O}\right\}_{\infty}$ (2) have been obtained attempting to modify a prototype truly molecular cooler with ferromagnetic interactions. The structures of both compounds as determined by single-crystal X-ray diffraction are reported, together with a detailed study of their magnetic and thermal properties, describing for both compounds a large magneto-caloric effect (MCE). The dominant ferromagnetic interaction present in $\mathbf{2}$ clearly favours this material for low applied magnetic fields, with respect to $\mathbf{1}$, that exhibit antiferromagnetic interactions. Magneto-structural correlations are derived for the sign and strength of the magnetic interactions in carboxylato-bridged Gd(III).

\section{Introduction}

Certain molecular or molecular-based compounds are recognized as good candidates for magnetic refrigeration for liquid-helium temperatures. ${ }^{1}$ This is because of the large magnetocaloric effect (MCE) exhibited by these materials at temperatures typically below $10 \mathrm{~K}$, i.e., a large variation of magnetic entropy $\left(\Delta S_{\mathrm{m}}\right)$ and adiabatic temperature $\left(\Delta T_{\mathrm{ad}}\right)$ induced by a magnetic field change. ${ }^{1,2}$ As for conventional cryogenic magnetic refrigerants, e.g., Gadolinium Gallium Garnet (GGG), ${ }^{3}$ these so-called molecular coolers usually contain the isotropic Gd(III) ion because it has zero orbital angular momentum and provides the largest entropy per single ion. Although both homo- and heterometallic high nuclearity complexes have been reported to have high MCE,, ${ }^{4,5}$, small lightweight Gd(III) compounds represent the best compromise in terms of weak magnetic interactions, magnetic/non-magnetic elements ratio and spin multiplicity. ${ }^{7,8}$ Enhancement of the latter through intramolecular ferromagnetic exchange is of course welcome, as it helps reaching large magnetic entropy at comparatively lower applied magnetic fields. A good example of these considerations is provided by gadolinium acetate tetrahydrate, $\left[\mathrm{Gd}_{2}\left(\mathrm{CH}_{3} \mathrm{CO}_{2}\right)_{6}\left(\mathrm{H}_{2} \mathrm{O}\right)_{4}\right] \cdot 4 \mathrm{H}_{2} \mathrm{O}$ (Gd-ac in the following), whose exceptional MCE remains among the most appealing reported thus far for a truly molecular material. ${ }^{1 \mathrm{c}, 7}$

Because the important parameter for application is the volumetric $\Delta S_{\mathrm{m}}, \mathrm{Gd}(\mathrm{III})$ metal-organic frameworks of various dimensionalities obtained with light and short bridging ligands have more recently provided significant improvements of MCE capacities. ${ }^{9}$ Indeed, as long as magnetic ordering is avoided down to the target working temperature, the dimensionality of the refrigerant material has no effect, ${ }^{10}$ while potentially giving access to high intrinsic density. With its sub-Kelvin ordering temperature and huge MCE, gadolinium formate actually compares favourably in terms of Relative Cooling Power $\left(\mathrm{RCP}^{2}\right)$ with the commercially exploited GGG. ${ }^{11}$

These recent results leave only small improvements of the actual MCE of molecular coolers left to be made, ${ }^{12}$ and we aim here at tackling two different though important aspects, i.e. on one hand avoid long-range magnetic order and on the other hand provide the molecular coolers a termination usable to make covalent attachment with surfaces. We focus on the prototype Gd-ac system that exhibits antiferromagnetic order around $0.2 \mathrm{~K}$, originating from an efficient packing and hydrogen bonding network involving lattice and coordinated water molecules. ${ }^{7}$ Its MCE properties have been shown to be maintained once deposited on $\mathrm{Si}$, although only through noncovalent interactions. ${ }^{13}$ Thus the coordinated water molecules and acetate ligands of Gd-ac have been replaced respectively by dimethylformamide and hydroxybutanoate, resulting in both cases in the formation of infinite coordination chains, i.e. compounds $\quad\left[\mathrm{Gd}\left(\mathrm{CH}_{3} \mathrm{CO}_{2}\right)_{3}(\mathrm{dmf})\right]_{\infty} \quad$ (1) and $\left.\left\{\left[\mathrm{Gd}\left(\mathrm{HO}\left(\mathrm{CH}_{2}\right)_{3} \mathrm{CO}_{2}\right)\right)_{3}\left(\mathrm{H}_{2} \mathrm{O}\right)\right] \cdot \mathrm{H}_{2} \mathrm{O}\right\}_{\infty}$ (2). Their single-crystal $\mathrm{x}$-ray structures, magnetic and thermal properties are reported, showing respectively weak antiferro- and ferromagnetic interactions, and large MCE in both cases. The opposite sign of the exchange interaction is discussed in light of magnetostructural considerations, and the resulting effect on the respective MCE is highlighted, especially at low applied fields. 


\section{Experimental}

\section{Synthesis}

Commercial $\mathrm{Gd}_{2} \mathrm{O}_{3}$ (99.9 \% trace metal basis, Aldrich), 4hydroxybutanoic acid lactone (or $\gamma$-butyrolactone, 98\% Aldrich) and analytical grade glacial acetic acid, N,N'dimethylformamide (dmf), absolute ethanol and diethylether were used without further purification, under aerobic conditions.

$\left[\mathrm{Gd}\left(\mathrm{CH}_{3} \mathrm{CO}_{2}\right)_{\mathbf{3}}(\mathrm{dmf})\right]_{\infty} \quad$ (1). Crystals of $\left[\mathrm{Gd}_{2}\left(\mathrm{CH}_{3} \mathrm{CO}_{2}\right)_{6}\left(\mathrm{H}_{2} \mathrm{O}\right)_{4}\right] \cdot 4 \mathrm{H}_{2} \mathrm{O}$ (100 mg, $\left.0.12 \mathrm{mmol}\right)$, prepared from $\mathrm{Gd}_{2} \mathrm{O}_{3}$ and acetic acid as described previously, ${ }^{7}$ were dissolved in dmf (20 mL), and the solution covered. Standing at room temperature of the colourless solution resulted after ca. 48 hours in the formation of colourless block/cube crystals of $\mathbf{1}$, suitable for X-ray crystallography. Crystals were recovered by filtration, washed with little dmf and dried in vacuo. The yield was $70 \%$ based on Gd (69 mg). Elemental analyses (wt. \%); calcd. for $\mathrm{C}_{9} \mathrm{H}_{16} \mathrm{GdNO}_{7}$ (found for 1): C 26.53 (26.4), H 3.96 (4.0), N 3.44 (3.5).

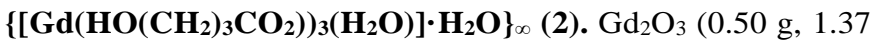
mmol) was added to a white suspension of excess 4hydroxybutanoic acid lactone (3.51 g, $40.8 \mathrm{mmol}$ ) in hot water (20 mL), and the resulting suspension stirred at $90{ }^{\circ} \mathrm{C}$ for 15 min. Aqueous $\mathrm{NaOH}(1 \mathrm{M}, 1 \mathrm{~mL})$ was then added resulting in a colourless solution after 1 hour at $90{ }^{\circ} \mathrm{C}$. The solution was filtered hot, and the filtrate left slowly evaporating. Colourless crystals of $\mathbf{2}$ suitable for X-ray crystallography formed when the remaining volume was $<1 \mathrm{~mL}$. Bulk white polycrystalline $\mathbf{2}$ is obtained upon further evaporation to almost dryness. The bulk solid was recovered and filtrated with an EtOH:diethylether 1:4 mixture, washed with little cold water and dried in vacuo. The total yield was $50 \%$ based on $\mathrm{Gd}$ (690 mg). Elemental analyses (wt. \%); calcd. for $\mathrm{C}_{12} \mathrm{H}_{25} \mathrm{GdO}_{11}$ (found for 2): C 28.68 (28.6), 5.01 (4.9).

\section{Physical measurements}

Infra-red spectra of neat samples were taken on a Perkin-Elmer Spectrum 100 apparatus equipped with an ATR device. Elemental analyses were performed by the Servei de Microanalisi, Consejo Superior de Investigaciones Cientifícas (CSIC) of Barcelona. Magnetic measurements were performed on bulk microcrystalline powders using a commercial SQUID magnetometer of the Physical Measurements unit of the Servicio General de Apoyo a la Investigación-SAI, Universidad de Zaragoza. Correction for the experimentally measured contribution of the sample holder, and the sample diamagnetism, estimated from Pascal's tables, were applied. Heat capacities in the range $0.35-30 \mathrm{~K}$ were obtained using the relaxation method in a commercial ${ }^{3} \mathrm{He}$ set-up equipped with a 14 T magnet, also of the SAI Physical Measurements. Apiezon$\mathrm{N}$ grease was used to provide good internal thermal contact between the heater, thermometer and sample, in the form a pellet made out of bulk microcrystalline powders.
Data for compound $\mathbf{1}$ were obtained at $100 \mathrm{~K}$ on a colourless block using Mo $\mathrm{K}_{\alpha}$ radiation $(\lambda=0.7107 \AA$ ) on a Bruker APEX II QUAZAR diffractometer equipped with a microfocus multilayer monochromator, at the X-ray Diffraction unit of the Servicio General de Apoyo a la Investigación-SAI, Universidad de Zaragoza. Data reduction and absorption corrections were performed within CrysalisPro software. ${ }^{14}$ Data for compound 2 were collected on a colourless plate at $100 \mathrm{~K}$ with a Bruker APEX II CCD diffractometer on the Advanced Light Source beamline 11.3.1 at Lawrence Berkeley National Laboratory, from a silicon 111 monochromator $(\lambda=0.7749 \AA)$. Data reduction and absorption corrections were performed with SAINT and SADABS. ${ }^{16}$ Both structures were solved by direct methods and refined on $\mathrm{F}^{2}$ using the SHELX-TL suite. ${ }^{17}$ Crystallographic and refinement parameters are summarized in Table 1. All details can be found in the supplementary crystallographic data for this paper in cif format with CCDC numbers 971619 and 971620. These data can be obtained free of charge from The Cambridge Crystallographic Data Centre via www.ccdc.cam.ac.uk/data_request/cif.

\begin{tabular}{|c|c|c|}
\hline & 1 & 2 \\
\hline$T[\mathrm{~K}]$ & 100 & 100 \\
\hline Empirical formula & $\mathrm{C}_{9} \mathrm{H}_{16} \mathrm{GdNO}_{7}$ & $\mathrm{C}_{12} \mathrm{H}_{25} \mathrm{GdO}_{1}$ \\
\hline FW & 407.48 & 502.57 \\
\hline Wavelength $[\AA]]$ & 0.7107 & 0.7749 \\
\hline Crystal system & monoclinic & monoclinic \\
\hline Space group & $P 2_{1} / \mathrm{c}$ & $P 2_{1} / \mathrm{n}$ \\
\hline$a[\AA ̊]$ & $9.4084(5)$ & $6.2169(4)$ \\
\hline$b[\AA]$ & $17.0945(9)$ & $10.5404(6)$ \\
\hline$c[\AA]$ & $8.1850(4)$ & $25.9134(15)$ \\
\hline$\beta\left[^{\circ}\right]$ & 93.862(2) & $92.920(1)$ \\
\hline$V\left[\AA^{3}\right]$ & 1313.42(12) & $1695.86(18)$ \\
\hline $\mathrm{Z}$ & 4 & 4 \\
\hline$\rho\left[\mathrm{g} / \mathrm{cm}^{3}\right]$ & 2.061 & 1.868 \\
\hline Reflections & 3388 & 4728 \\
\hline Parameters & 168 & 226 \\
\hline Restraints & 0 & 8 \\
\hline$R_{\text {int }}$ & 0.0252 & 0.0484 \\
\hline$R_{1}[\mathrm{I}>2 \sigma(\mathrm{I})]^{[\mathrm{a}]}$ & 0.0167 & 0.0331 \\
\hline $\mathrm{w} R_{2}[\mathrm{I}>2 \sigma(\mathrm{I})]^{[\mathrm{b}]}$ & 0.0397 & 0.0720 \\
\hline$R_{1}$ [all data] $^{[\mathrm{a}]}$ & 0.0178 & 0.0449 \\
\hline $\mathrm{w} R_{2}$ [all data] ${ }^{[\mathrm{b}]}$ & 0.0405 & 0.0761 \\
\hline Goodness-of-fit & 1.091 & 1.055 \\
\hline
\end{tabular}

\section{X-ray crystallography}



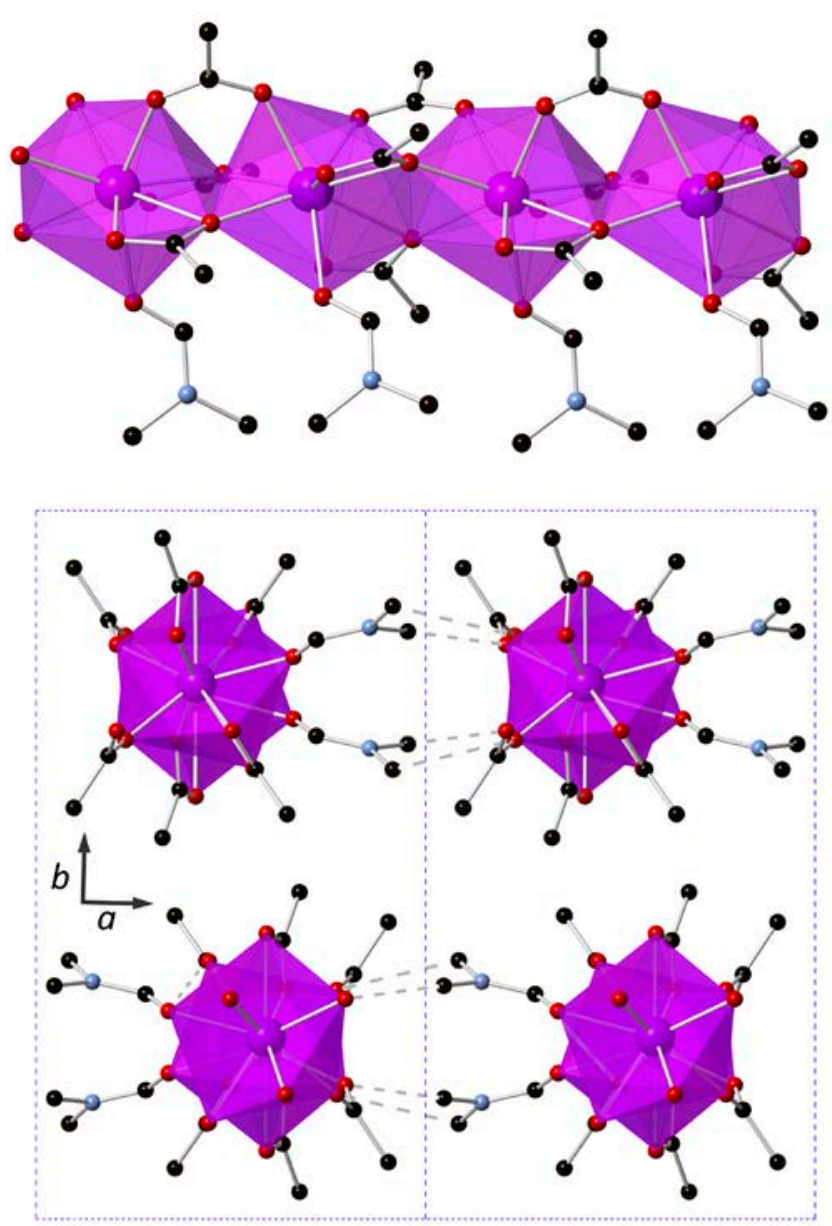

Figure 1. Top: A view of the coordination chains in 1, propagating along the $c$ axis. Bottom: View of the packing along the chain axis, showing interchain $\mathrm{C}-$ $\mathrm{H} \cdots \mathrm{O}$ contacts as dashed grey lines and the cell limits as light blue dashed lines. Colour code: purple, Gd; red, O; light blue, N; black C. Hydrogens are omitted for clarity.

\section{Results and Discussion}

\section{Synthesis and crystal structures}

Among truly molecular coolers, $\left[\mathrm{Gd}_{2}\left(\mathrm{CH}_{3} \mathrm{CO}_{2}\right)_{6}\left(\mathrm{H}_{2} \mathrm{O}\right)_{4}\right] \cdot 4 \mathrm{H}_{2} \mathrm{O}$ or $\mathrm{Gd}_{2}$-ac remains an optimal material, resulting from high density and one of the largest ferromagnetic interaction within Gd(III) pairs. ${ }^{7}$ However, the dense packing of molecules through hydrogen bonds results in a dipolar magnetic order around $0.2 \mathrm{~K}$, impeding cooling to lower temperatures. In order to destroy this magnetic order or bring it to lower temperatures while maintaining the dinuclear core of $\mathrm{Gd}_{2}$-ac, we attempted replacing the coordinated and lattice water molecules of $\mathrm{Gd}_{2}$-ac by molecules less prone to intermolecular interactions. We thus recrystallized $\mathrm{Gd}_{2}$-ac from $\mathrm{dmf}$ solution, resulting in the formation of cube-shape colourless crystals of $\left[\mathrm{Gd}\left(\mathrm{CH}_{3} \mathrm{CO}_{2}\right)_{3}(\mathrm{dmf})\right]_{\infty}(\mathbf{1})$. Having successfully replaced water molecules and maintained similar Gd vs. non-magnetic weight ratio and density, the synthetic system has however evolved into coordination chains. A similar chain compound had been previously formed by reaction of $\mathrm{Gd}_{2}$-ac with acetylacetone in methanol, confirming a tendency to form extended structures. ${ }^{17}$ Compound 1 crystallizes in the monoclinic $P 21_{1}$ /c space group with an asymmetric unit comprising a $\mathrm{Gd}(\mathrm{III})$ metal ion coordinated by three acetate ions and a terminal dmf molecule. The unique $\mathrm{Gd}$ site is nine-coordinated with $\mathrm{Gd}-\mathrm{O}$ bond distances ranging $2.3473(14)$ to $2.5445(16) \AA$ and forming a capped square antiprism coordination sphere. The structure is solely built on chains running along the $c$ axis (Figure 1 top), in which the neighbouring $\mathrm{Gd}(\mathrm{III})$ ions are bridged by two $\mu \mathrm{O}: \kappa^{2} \mathrm{OO}$ and one $\mu \mathrm{OO}$ syn-syn acetate groups, with $\mathrm{Gd} \cdots \mathrm{Gd}$ separation of $4.100 \AA$. The $\mu \mathrm{O}: \kappa^{2} \mathrm{OO}$ acetate groups are chelating the same $\mathrm{Gd}(\mathrm{III})$ ion, as opposed to $\mathrm{Gd}_{2}$-ac or the similar chain with methanol as terminal ligand ${ }^{17}$ in which the bridges are symmetric. To the best of our knowledge, this asymmetric topology of the bridge in $\mathbf{1}$ is unprecedented in carboxylate-based Gd(III) compounds. ${ }^{18}$ The bridge through oxygen $\mathrm{O} 3$ has rather unequal $\mathrm{Gd}-\mathrm{O}$ distances, at 2.3833(14) and 2.5238(13) $\AA$, while those through $\mathrm{O} 1$ are similar, i.e. 2.4467(14) and 2.4666(14) $\AA$, and so are the Gd-O-Gd angles, at $113.12(6)$ and $113.30(6)^{\circ}$ respectively. The successive $\mathrm{Gd}_{2} \mathrm{O}_{2}$ bridges are tilted by $59.8^{\circ}$, but the chain remains quite linear with a $\mathrm{Gd} \cdots \mathrm{Gd} \cdots \mathrm{Gd}$ angle of $174.15^{\circ}$, the $\mathrm{dmf}$ molecules pointing towards approximately the same direction. Indeed the methyl groups of these dmf molecules make a relatively short $\mathrm{C}-\mathrm{H} \cdots \mathrm{O}$ contact with the neighbouring chain along the $a$ axis (Figure 1 bottom). This is actually the only significant interchain interaction in the crystal packing, confirming the better isolation obtained through the dmf molecules. Shortest interchain $\mathrm{Gd} \cdots \mathrm{Gd}$ separations are 9.408, i.e. the $a$ axis vector, and $8.732 \AA$ along the $b$ axis. Adjacent chains along the $b$ axis direction are rotated by $180^{\circ}$ with respect to the chain axis $c$ (Figure 1 bottom).

Molecular coolers such as Gd-ac are candidates to provide on-chip devices efficient local refrigeration, ${ }^{13}$ but for this purpose a strong interaction of the molecules to the surface, ideally through covalent binding, is required. We therefore attempted to make a compound similar to Gd-ac, using a carboxylic acid with a termination allowing further reactivity. Hydroxy-butanoic acid was considered a good candidate for this, in particular because the butyl arm would provide some flexibility, while still maintaining a reasonably high Gd vs. non magnetic elements weight ratio. The reaction of excess 4hydroxybutanoic acid lactone, a precursor of hydroxy-butanoic acid, with gadolinium oxide in aqueous basic conditions resulted in the formation of another coordination chain compound, $\left.\quad\left\{\left[\mathrm{Gd}\left(\mathrm{HO}\left(\mathrm{CH}_{2}\right)_{3} \mathrm{CO}_{2}\right)\right)_{3}\left(\mathrm{H}_{2} \mathrm{O}\right)\right] \cdot \mathrm{H}_{2} \mathrm{O}\right\}_{\infty}$ Considering that a similar reaction using pentanoic acid resulted in a dinuclear complex, ${ }^{19}$ the probable factor favouring the chain propagation in $\mathbf{2}$ is the ability of the flexible hydroxybutyl group to bend and form intrachain hydrogen bonding (see below). 


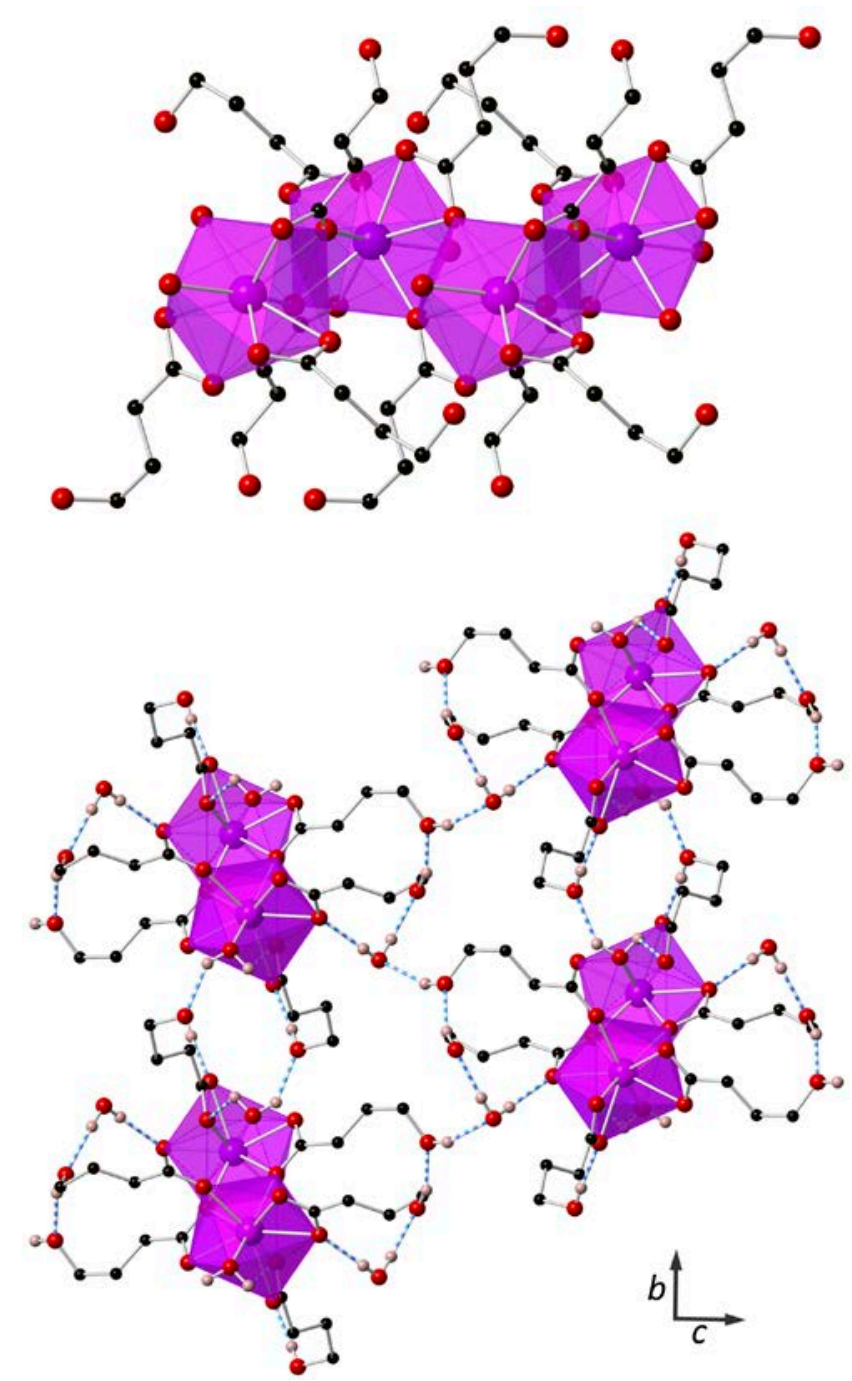

Figure 2. Top: A view of the zig-zag coordination chains in 2, propagating along the $a$ axis. Hydrogens are omitted for clarity. Bottom: View of the packing along the chain axis, showing interchain hydrogen bonds as dashed blue sticks. Colour code: purple, Gd; red, O, light blue; N; black, C; beige, H. Only hydrogens involved in $\mathrm{H}$-bonds are shown.

Compound 2 crystallizes in the monoclinic $P 2_{1} / \mathrm{n}$ space group with an asymmetric unit comprising a unique Gd(III) site coordinated by two bridging and one terminal chelating hydroxybutanoate ions and an aqua ligand and a lattice water molecule. The extended chain structure along the $a$ axis (Figure 2 top) builds on two very similar successive symmetric double $\mu \mathrm{O}: \kappa^{2} \mathrm{OO}$ carboxylic bridges with Gd $\cdots$ Gd separations of 4.140 and 4.083 $\AA$, Gd-O-Gd angles of $113.70(10)$ and 113.05(10) and $\mathrm{Gd}-\mathrm{O}$ bond distances of 2.411(3)/2.533(3) 2.374(3)/2.520(3) $\AA$, respectively through atoms O5 and O7. Interestingly, these bridge topology and characteristics are similar to those in $\mathrm{Gd}_{2}$-ac (4.183 $\AA, 115.31^{\circ}, 2.393 / 2.558 \AA$ ). The successive $\mathrm{Gd}_{2} \mathrm{O}_{2}$ planes are tilted by $87.4^{\circ}$, resulting in a

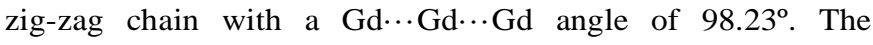
chelating third pentanoate carboxylic group and terminal water molecule complete the nine-coordinate environment of the Gd site, distorted capped square antiprism close to a "muffin”, with
Gd-O bond distances ranging 2.357(3) to 2.533(3) $\AA$. This third hydroxybutyl group is bent in the chain direction and its hydroxyl oxygen $\mathrm{O} 3$ forms an intrachain hydrogen bond with the carboxylic oxygen O2. The other two hydroxybutyl, although elongated in the $c$ axis direction, also form an intrachain hydrogen bond between their respective hydroxyl groups $\mathrm{O} 6$ and O9. The coordinated water molecule $\mathrm{O} 10$ also forms an intrachain hydrogen bond with the neighbouring bridging $\mathrm{O} 1$ atom. The lattice water molecule $\mathrm{O} 1 \mathrm{~W}$ also participates of this dense intrachain hydrogen bonding network (Figure 2 bottom), interacting as donor with the hydroxyl O6 and the carboxylic $\mathrm{O} 4$ oxygens. The shortest interchain Gd $\cdots$ Gd separation is $8.498 \AA$, in the $b$ axis direction, arising from the only direct interchain interaction through hydrogen bonding of the coordinated water molecule $\mathrm{O} 10$ to the bent hydroxybutyl of two neighbouring chains. Chains also interact in the $c$ axis direction, although through the lattice water molecule that also forms, as acceptor, a hydrogen bond with the hydroxyl O9 atom. Each chain is thus connected to four additional neighbours, rotated by $23.35^{\circ}$ with respect to the chain axis $a$, with longer interchain $\mathrm{Gd} \cdots \mathrm{Gd}$ separation of $13.487 \AA$ (Figure 2 bottom). Details of this hydrogen bonding network in the structure of $\mathbf{2}$ are given in Table 2 .

Table 2. Distance and angles describing the hydrogen bonding network in the structure of $\left.\left\{\left[\mathrm{Gd}\left(\mathrm{HO}\left(\mathrm{CH}_{2}\right)_{3} \mathrm{CO}_{2}\right)\right)_{3}\left(\mathrm{H}_{2} \mathrm{O}\right)\right] \cdot \mathrm{H}_{2} \mathrm{O}\right\}_{\infty}(2)$.

\begin{tabular}{|c|c|c|c|c|}
\hline $\mathrm{D}-\mathrm{H} \cdots \mathrm{A}$ & D-H $(\AA)$ & $\mathrm{H} \cdots \mathrm{A}(\AA)$ & $\mathrm{D} \cdots \mathrm{A}(\AA)$ & $\mathrm{D}-\mathrm{H}-\mathrm{A}\left(\left(^{\circ}\right)\right.$ \\
\hline 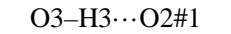 & 0.84 & 1.87 & $2.713(4)$ & 174.9 \\
\hline 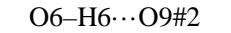 & $0.890(17)$ & $1.880(17)$ & $2.731(5)$ & $159(5)$ \\
\hline O10-H10D $\cdots \mathrm{O} 1 \# 2$ & 0.89 & 2.37 & $2.738(4)$ & 104.9 \\
\hline $\mathrm{O} 1 \mathrm{~W}-\mathrm{H} 2 \mathrm{~W} \cdots \mathrm{O} 4$ & $0.888(19)$ & $1.98(2)$ & $2.846(4)$ & $164(5)$ \\
\hline O1W-H1W $\cdots$ O6\#1 & $0.904(19)$ & 1.93(3) & $2.822(5)$ & 171(5) \\
\hline O10-H10C $\cdots$ O3\#3 & 0.89 & 1.91 & $2.657(4)$ & 141.6 \\
\hline O9-H9 ‥ O1W\#4 & 0.84 & 1.93 & $2.720(5)$ & 155.3 \\
\hline
\end{tabular}

Symmetry operations: \#1: 1+x,y,z; \#2: -1+x,y,z; \#3: 1-x,1-y,-z; \#4: 1/2-x,$1 / 2+y, 1 / 2-z$.

\section{Magnetic and thermal properties}

The variable-temperature magnetic properties of $\mathbf{1}$ and $\mathbf{2}$ are shown in the form of $\chi T$ vs. $T$ plot, $\chi$ being the molar magnetic susceptibility derived from direct current (dc) magnetization collected in the 2-100 $\mathrm{K}$ temperature range and in an applied field of $0.01 \mathrm{~T}$ (Figure 3). For both compounds, the $\chi T$ value at $100 \mathrm{~K}$ is close to that expected for a spin-only $s=7 / 2 \mathrm{Gd}(\mathrm{III})$ ion $\left(7.875 \mathrm{~cm}^{3} \mathrm{Kmol}^{-1}\right)$. First, $\chi T$ stays roughly constant as the temperature is decreased, and at approximately $20 \mathrm{~K}$ a decrease/increase sets in, reaching a minimum/maximum of approximately $6.0 / 9.3 \mathrm{~cm}^{3} \mathrm{Kmol}^{-1}$ at $2 \mathrm{~K}$, respectively for $\mathbf{1} / \mathbf{2}$. These behaviours point at weak magnetic interactions of opposite signs, antiferromagnetic in $\mathbf{1}$ while ferromagnetic in $\mathbf{2}$. This is confirmed by isothermal magnetizations vs. field at $2 \mathrm{~K}$ that are slightly below and above the Brillouin function for $s=$ $7 / 2$ and $g=2$ (Fig. S1), respectively for 1 and 2 . The fits of the temperature-dependencies of the susceptibility to the CurieWeiss law, $1 / \chi=C /\left(T-\theta_{\mathrm{w}}\right)$ (Fig. S2) provide the same Curie constant $C \approx 7.88 \mathrm{~cm}^{3} \mathrm{Kmol}^{-1}$ for both complexes, as expected 
for $\mathrm{Gd}(\mathrm{III})$ ion, and the Weiss constants $\theta_{\mathrm{W}}=-0.5 \mathrm{~K}$ and 0.4 $\mathrm{K}$ for $\mathbf{1}$ and 2, respectively. The different signs for $\theta_{\mathrm{w}}$ are a further corroboration of the type of dominant interaction involved: antiferromagnetic (1) and ferromagnetic (2).

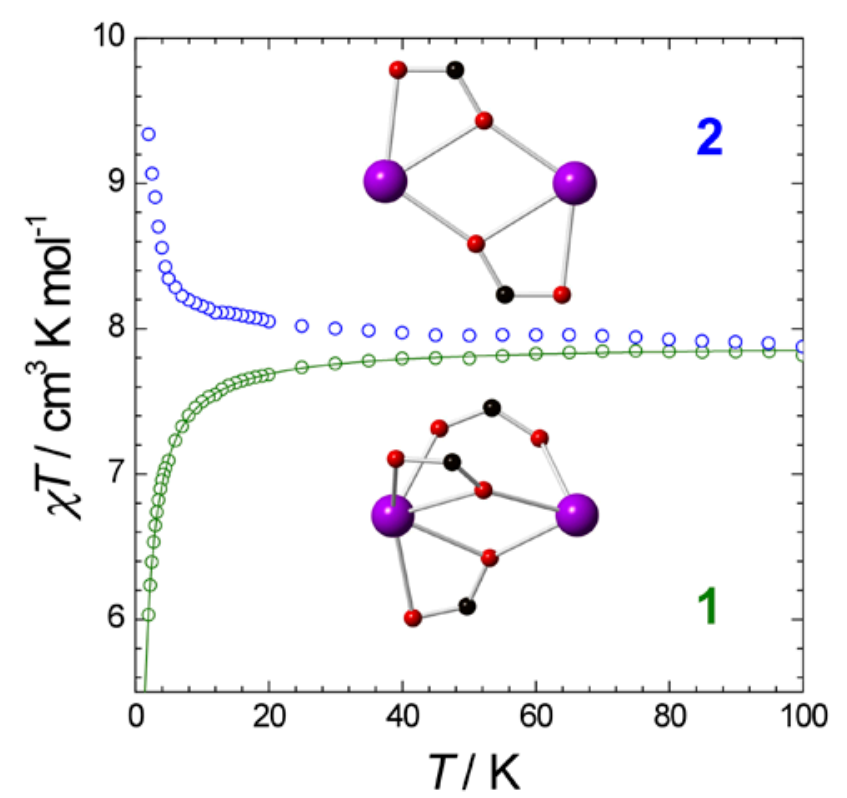

Figure 3. Temperature-dependencies $(2-100 \mathrm{~K})$ of the dc molar susceptibility for $\mathbf{1}$ (blue circles) and $\mathbf{2}$ (green circles) at $0.01 \mathrm{~T}$ applied field. Full line is the best-fit to a $s=7 / 2$ chain model for 1 , see main text.

The molar specific heat for $\mathbf{1}$ and $\mathbf{2}$ is reported in Figure 4 for selected applied fields. The lattice contribution, $C_{\text {latt, }}$ is modelled (dashed lines) using the Debye function, which yields the characteristic Debye temperatures $\theta_{\mathrm{D}}=65.6 \mathrm{~K}$ and $77.7 \mathrm{~K}$ for 1 and 2, respectively.

For 2, a prominent lambda-like anomaly in the zero-field specific heat occurs at $T_{C}=0.45 \mathrm{~K}$, denoting a phase transition, whose magnetic origin is proved since this feature disappears on applying a magnetic field. The peak occurs at a temperature comparable to $\theta_{\mathrm{w}}$, suggesting that interactions propagate threedimensionally without a preferred direction. The mean-field expression for the transition temperature, $\theta_{\mathrm{W}}=z|J| s(s+1) / 3 k_{\mathrm{B}}$, can be employed to obtain $z J / k_{\mathrm{B}}=0.052 \mathrm{~cm}^{-1}$, where $z$ is the number of nearest magnetic neighbours.

In the case of $\mathbf{1}$, no phase transition is detected down to the lowest experimentally-accessible temperature. Thus, we tentatively associate the observed magneto-thermal properties to $1 \mathrm{D}$ magnetic fluctuations within the chains, depicted in Figure 1. We model the experimental susceptibility of $\mathbf{1}$ by using Fisher's expression derived for a Heisenberg chain of $s=$ $7 / 2$ spins and a Hamiltonian of type $-J \sum S_{\mathrm{i}} \cdot S_{\mathrm{i}+1} \cdot{ }^{20}$ The full line in Figure 3 shows the excellent agreement that is obtained for $g$ $=2.00$ and $J / k_{\mathrm{B}}=-0.035 \mathrm{~cm}^{-1}$. It is worth mentioning that the same model applied to 2 provides a poor description of the $\chi$ data, ${ }^{21}$ hence validating our aforementioned approach for this complex.

The specific heat measurements in applied fields, $B_{0}$, are characterized by Schottky-type anomalies (Figure 4), that we analyse as follows. In a mean-field approach, the onset of exchange interactions can be interpreted in terms of an interaction field $B_{\text {int }}$, producing a Zeeman splitting of the (otherwise degenerate) Gd(III) spin-multiplet and, thus, leading to a Schottky-type anomaly, as observed. When measuring the specific heat in $B_{0}$, the total field becomes the sum of $B_{\text {int }}$ and $B_{0}$, so the anomaly should shift to higher or lower temperatures,

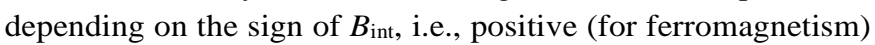
or negative (antiferromagnetism), respectively. We calculate the Schottky-type anomalies for fields $B_{0}+B_{\text {int }}$, where $B_{\text {int }}$ is introduced as a free parameter. The best agreement with the experimental data is obtained for $B_{\text {int }}=-0.15 \mathrm{~T}$ and $0.3 \mathrm{~T}$ for $\mathbf{1}$ and 2, respectively (solid lines in Fig. 4), as expected because of the ferromagnetic interactions in $\mathbf{1}$ and antiferromagnetic ones in $\mathbf{2}$.

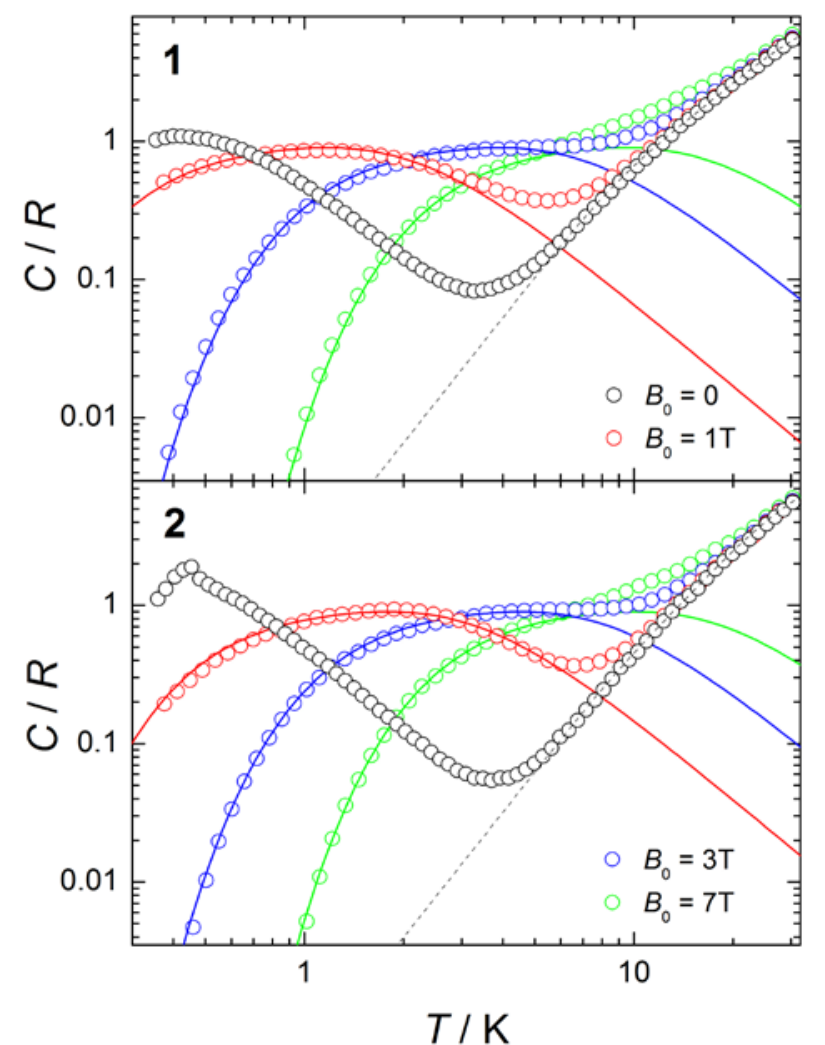

Figure 4. Temperature-dependencies $(0.3-30 \mathrm{~K})$ of the specific heat, normalized to $\mathrm{Gd}(\mathrm{III})$ ions and gas constant $R$, for 1 (top panel) and 2 (bottom panel), collected for $B_{0}=0,1,3$ and $7 \mathrm{~T}$, as labelled. The in-field data are well reproduced by Schottky calculations (solid lines) for $s=7 / 2$ in presence of an additional interaction field $B_{\text {int }}$. Lattice contributions are reported as dashed lines.

Using the specific heat data, we evaluate the magnetic entropy according to the expression $S_{m}(T)=\int_{C_{m}}(T) / T \mathrm{~d} T$, where $C_{\mathrm{m}}$ is the magnetic contribution to the specific heat, which we obtain by subtracting $C_{\text {latt }}$ to the total heat capacity. The soderived $S_{\mathrm{m}}(T, B)$ curves for both complexes are depicted in Figure S3. Next, we evaluate the MCE, specifically, the magnetic entropy change $-\Delta S_{\mathrm{m}}(T, \Delta B)=S_{\mathrm{m}}\left(B_{\mathrm{f}}\right)-S_{\mathrm{m}}\left(B_{\mathrm{i}}\right)$ for the 
magnetic field change $\Delta B=B_{\mathrm{f}}-B_{\mathrm{i}}$. The so-obtained results are depicted in Figure 5, together with $\Delta S_{\mathrm{m}}(T, \Delta B)$ that we derive by applying the Maxwell relation, $\Delta S_{\mathrm{m}}(T, \Delta B)=\int[\partial M(T, B) / \partial T]_{B} \mathrm{~d} B$, to the magnetization, $M(T, B)$, data (Fig. S4). The nice agreement proves the validity of both independent approaches. For both molecules, the MCE reaches large values that are comparable with that reported in the recent literature for Gd(III)-based complexes..$^{1,4-5,7-8}$ Figure 5 shows that, for $\Delta B=$ $7 \mathrm{~T},-\Delta S_{\mathrm{m}}$ reaches $38.8 \mathrm{Jkg}^{-1} \mathrm{~K}^{-1}$ for $\mathbf{1}$ and $31.8 \mathrm{Jkg}^{-1} \mathrm{~K}^{-1}$ for 2 at $T=1.8 \mathrm{~K}$. It is worth mentioning that, if the entropy change is reported in mass unit, then the entropy change on increasing $\Delta B$ tends to larger values in $\mathbf{1}$, because of its relatively lighter molecular mass (by 20\%). However, for the smaller field changes (e.g., $\Delta B=1 \mathrm{~T}$ ), the MCE is chiefly driven by the magnetic interactions. Therefore, under these conditions, $-\Delta S_{\mathrm{m}}$ is larger in $\mathbf{2}$ since antiferromagnetic interactions are detrimental to the MCE. ${ }^{2 b}$ This can be clearly evidenced by reporting the entropy changes in molar unit and normalized to Gd(III), see Figure S5.

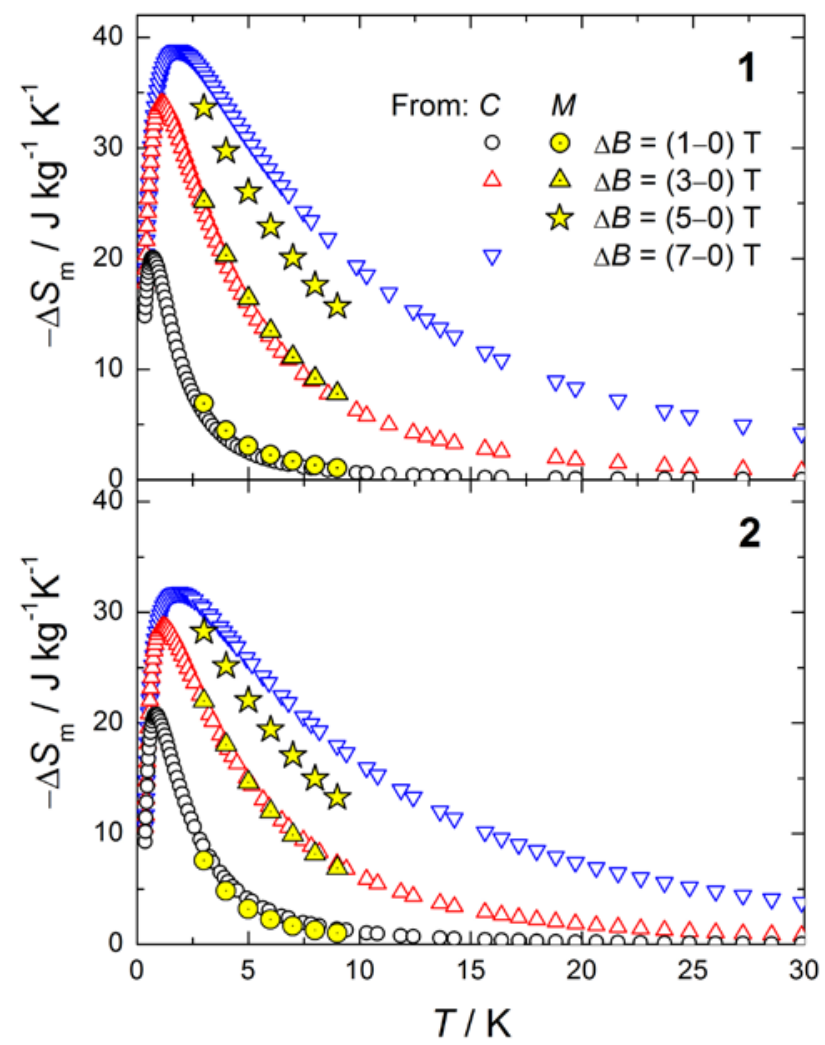

Figure 5. Temperature-dependencies of the magnetic entropy change, $\Delta S_{\mathrm{m}}$, as obtained from specific heat data, for the indicated applied-field changes $\Delta B$, for 1 (top) and 2 (bottom). The corresponding $\Delta S_{m}$ values derived from magnetization data are shown as full markers for $\Delta B$ of 1,3 and $5 \mathrm{~T}$.

\section{Magneto-structural considerations}

Magnetic exchange coupling in compounds with Gd(III) ions connected through oxygen donors has been studied extensively, in particular for compounds with various types of bridge topologies based on carboxylate ligands. ${ }^{7,17,19,22-25}$ However, no clear correlation of any specific structural parameter with the magnitude and sign of the exchange coupling has yet been found. ${ }^{19,22}$ This is probably due in part to the weakness of the exchange couplings, together with the variety of bridge topologies encountered. Indeed, for a variety of compounds with bridge topologies involving different bridging modes of carboxylate groups, so-called $\mathrm{B}$ - and C-types i.e. two symmetric $\mu \mathrm{O}: \kappa^{2} \mathrm{OO}$ and respectively two or one $\mu \mathrm{OO}$ syn-syn carboxylates, ${ }^{19,23}$ antiferromagnetic and ferromagnetic interactions of varying strengths have been reported. ${ }^{19,22}$ There is though one bridge topology for which a systematic trend seems to hold. For compounds with Gd(III) pairs of the socalled A-type, i.e. solely bridged by two $\mu \mathrm{O}: \kappa^{2} \mathrm{OO}$ carboxylates, a ferromagnetic coupling is in most cases observed. ${ }^{7,19,23-24}$ This trend is confirmed here with the weak ferromagnetic interaction derived in $\mathbf{2}$. Actually, the magnitude of the interaction in these compounds does correlate with the Gd $\cdots$ Gd separation, although not linearly, as shown in Figure 6 (details in Table S1). For the shortest separation, the antiferromagnetic contribution dominates, but the balance seems to be very subtle, with a very steep transition towards dominant ferromagnetic interactions for only slightly larger separations. The interaction rapidly reaches a maximum around $4.19 \AA$, and then decreases smoothly with increasing $\mathrm{Gd} \cdots \mathrm{Gd}$ distances. Interestingly, the prototype molecular cooler Gd-ac seems to represent the optimal structural configuration for a maximal ferromagnetic exchange. It should be noted that the $\mathrm{Gd}-\mathrm{O}-\mathrm{Gd}$ angle remains in all cases above $112.5^{\circ}$. More acute angles and shorter Gd $\cdots$ Gd separations are likely not accessible with this bridge topology since then additional syn-syn carboxylates are favoured. This is clearly shown by comparing with compounds with a B-type bridge (Figure S6 right). ${ }^{22,25}$ These all present Gd-O-Gd angles below $110^{\circ}$, and the corresponding set of (mostly antiferromagnetic) interaction constants also correlates reasonably, in this case linearly, with the $\mathrm{Gd} \cdots \mathrm{Gd}$ separation (Figure S6 left). It is interesting to note that the approximate critical value of $\mathrm{Gd} \cdots \mathrm{Gd}$ distance for which the coupling changes sign is similar for both groups of compounds, at ca. $4.12 \AA$. As originally postulated by Perec et al., ${ }^{23}$ these separate correlations found for two types of carboxylate-bridged Gd(III) pairs seem to indicate that more acute Gd-O-Gd and shorter Gd*G distance favour the antiferromagnetic contribution to the exchange coupling, through a likely increase of orbital overlap either through the oxygen bridge and/or through space. While these correlations could be used to optimize the MCE of Gd(III)-carboxylate compounds (see Fig. S5 and the aforementioned discussion on the sign of the interaction), it is however obviously difficult to control synthetically the Gd $\cdots$ Gd separation or even the type of bridge formed by carboxylate ligands. 


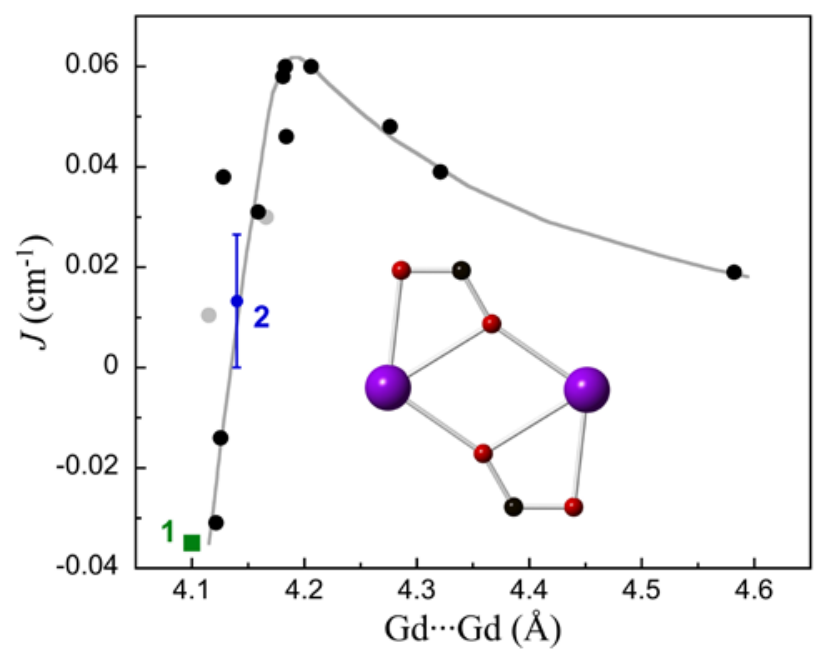

Figure 6. Correlation of the exchange coupling in $\mathrm{Gd}(\mathrm{III})$ pairs bridged by either two $\mu \mathrm{O}: \kappa^{2} \mathrm{OO}$ carboxylates (A-type, black dots) vs. the $\mathrm{Gd} \cdots \mathrm{Gd}$ separation. Grey dots are compounds with two different di $\left(\mu \mathrm{O}: \kappa^{2} \mathrm{OO}\right.$ carboxylate) bridges for which average values of the $\mathrm{Gd} \cdots \mathrm{Gd}$ separation and $\mathrm{Gd}-\mathrm{O}-\mathrm{Gd}$ angle have been used. The full line is a guide for the eye highlighting the reasonable correlation found. The range given for 2 derives from $z J / k_{\mathrm{B}}=0.052 \mathrm{~cm}^{-1}$ (see text) and from assuming $2<z<6$.

\section{Conclusions}

Aiming to modify $\left[\mathrm{Gd}_{2}\left(\mathrm{CH}_{3} \mathrm{CO}_{2}\right)_{6}\left(\mathrm{H}_{2} \mathrm{O}\right)_{4}\right] \cdot 4 \mathrm{H}_{2} \mathrm{O}$, a prototype truly molecular cooler with ferromagnetic interactions, in order to either suppress its magnetic order or provide it with chemical functions usable for covalent grafting to surfaces, the infinite coordination chain compounds $\left[\mathrm{Gd}\left(\mathrm{CH}_{3} \mathrm{CO}_{2}\right)_{3}(\mathrm{dmf})\right]_{\infty}$ (1) and $\left.\left\{\left[\mathrm{Gd}\left(\mathrm{HO}\left(\mathrm{CH}_{2}\right)_{3} \mathrm{CO}_{2}\right)\right)_{3}\left(\mathrm{H}_{2} \mathrm{O}\right)\right] \cdot \mathrm{H}_{2} \mathrm{O}\right\}_{\infty}$ (2) have been formed. Replacement of coordinated water molecules by dimethylformamide in $\mathbf{1}$ does impede the presence of a magnetic order due to the absence of intermolecular interactions, but modification of the acetate bridging mode results in switching the magnetic interaction to antiferromagnetic. Replacing acetate ligands by hydroxybutanoate in $\mathbf{2}$ does succeeds in providing a ferromagnetically-coupled system with appended hydroxybutyl groups. Magneto-thermal studies indicate a large magnetocaloric effect for both compounds, although the effect of the opposite sign of the exchange interaction is shown to favour $\mathbf{2}$ at low applied fields, compensating its lower magnetic/nonmagnetic elements ratio. The magnetic interactions at work in both compounds have been discussed in light of magnetostructural correlations, that indicate the original $\left[\mathrm{Gd}_{2}\left(\mathrm{CH}_{3} \mathrm{CO}_{2}\right)_{6}\left(\mathrm{H}_{2} \mathrm{O}\right)_{4}\right] \cdot 4 \mathrm{H}_{2} \mathrm{O}$ material possesses optimal structural parameters, at least among carboxylato-bridged systems. We will now aim to use $\mathbf{2}$ and similar systems to functionalize surfaces and thus advance towards true on-chip cooling with efficient cryogenic molecular coolers.

\section{Acknowledgements}

This work has been supported by the Spanish MINECO and FEDER through grants MAT2011-24284 and MAT201238318-C03 and by an EU Marie Curie IEF (PIEF-GA-2011299356 to G. L.). The Advanced Light Source is supported by the Director, Office of Science, Office of Basic Energy
Sciences of the U.S. Department of Energy under contract no. DE-AC02-05CH11231.

\section{Notes and references}

a Instituto de Ciencia de Materiales de Aragón (ICMA), CSIC and Universidad de Zaragoza, Plaza San Francisco s/n, 50009 Zaragoza, Spain. Fax: 34976 761229; Tel: 34976 762461; E-mail: roubeau@unizar.es.

$b$ Advanced Light Source, Berkeley Laboratory, 1 Cyclotron Road, Berkeley, CA 94720, USA.

$\dagger$ Footnotes should appear here. These might include comments relevant to but not central to the matter under discussion, limited experimental and spectral data, and crystallographic data.

Electronic Supplementary Information (ESI) available: [additional Figures presenting magnetic and thermal properties of $\mathbf{1}$ and 2, details of magneto-structural correlations data and additional correlations]. See DOI: $10.1039 / \mathrm{b} 000000 \mathrm{x} /$

1 a) M. Evangelisti and E. K. Brechin, Dalton Trans., 2010, 39, 46724676; b) R. Sessoli, Angew. Chem. Int. Ed., 2012, 51, 43-45; c) J. W. Sharples and D. Collison, Polyhedron, 2013, 54, 91-103

2 a) A. M. Tishin and Y. I. Spichkin, The Magnetocaloric Effect and its Applications, Taylor \& Francis, London 2003; b) M. Evangelisti, "Molecule-based magnetic coolers: measurement, design and application”, in Molecular Magnets. Physics and Applications, eds. F. Luis, J. Bartolomé and J. F. Fernandez, Springer-Verlag Berlin Heidelberg, 2014, pp 365-387

3 a) B. Daudin, R. Lagnier and B. Salce, J. Magn. Magn. Mater., 1982, 27, 315-322; b) T. Numazawa, K. Kamiya, T. Okano and K. Matsumoto, Physica B, 2003, 329-33, 1656-1657

4 high nuclearity $\left[\mathrm{Gd}_{\mathrm{x}}\right.$ ] complexes: a) J. W. Sharples, Y. Z. Zheng, F. Tuna, E. J. L. McInnes and D. Collison, Chem. Commun., 2011, 47, 7650-7652; b) R. J. Blagg, F. Tuna, E. J. L. McInnes and R. E. P. Winpenny, Chem. Commun., 2011, 47, 10587-10589; c) S-J. Liu, J-P. Zhao, J. Tao, J-M. Jia, S-D. Han, Y. Li, Y-C. Chen and X-H. Bu, Inorg. Chem., 2013, 52, 9163-9165; d) L-X. Chang, G. Xiong, L. Wang, P. Cheng and B. Zhao, Chem. Commun., 2013, 49, 10551057; F-S. Guo, Y-C. Chen, L-L. Mao, W-Q. Lin, J-D. Leng, R. Tarasenko, M. Orendác, J. Prokleska, V. Sechovsky and M-L. Tong, Chem. Eur. J., 2013, 19, 14876-14885

5 high nuclearity 3d-Gd complexes: a) with Mn see Y.-Z. Zheng, E. M. Pineda, M. Heliwell and R. E. P. Winpenny, Chem. Eur. J., 2012, 18, 4161-4165; with Cu see S. Langley, N. F. Chilton, B. Moubaraki, T. N. Hooper, E. K. Brechin, M. Evangelisti and K. S. Murray, Chem. Sci., 2011, 2, 1166-1169; c) with Co see Y-Z. Zheng, M. Evangelisti, F. Tuna and R. E. P. Winpenny, J. Am. Chem. Soc., 2012, 134, 10571065; d) with Cr see T. Birk, K. S. Pedersen, C. A. Thuesen, T. Weyhermüller, M. Schau-Magnussen, S. Piligkos, H. Weihe, S. Mossin, M. Evangelisti and J. Bendix, Inorg. Chem., 2012, 51, 54355443; e) with Ni see Y-Z. Zheng, M. Evangelisti and R. E. P. Winpenny, Angew. Chem. Int. Ed., 2011, 50, 3692-3695

6 3d complexes: a) R. Shaw, R. H. Laye, L. F. Jones, D. M. Low, C. Talbot-Eeckelaers, Q. Wei, C. J. Milios, S. Teat, M. Helliwell, J. Raftery, M. Evangelisti, M. Affronte, D. Collison, E. K. Brechin and E. J. L. McInnes, Inorg. Chem., 2007, 46, 4968-4978; b) M. Manoli, 
A. Collins, S. Parsons, A. Candini, M. Evangelisti and E. K. Brechin, J. Am. Chem. Soc., 2008, 130, 11129-11139

7 M. Evangelisti, O. Roubeau, E. Palacios, A. Camón, T. N. Hooper, E. K. Brechin and J. J. Alonso, Angew. Chem. Int. Ed., 2011, 50, 66066609

8 E. Colacio, J. Ruiz, G. Lorusso, E. K. Brechin and M. Evangelisti, Chem. Commun., 2013, 49, 3845-3847

9 a) L. Sedláková, J. Hanko, A. Orendácová, M. Orendác, C-L. Zhou, W-H. Zhu, B-W. Wang, Z-M. Wang and S. Gao, J. Alloys Compd., 2009, 487, 425-429; b) R. Sibille, T. Mazet, B. Malaman and M. François, Chem. Eur. J., 2012, 18, 12970-12973; c) F-S. Guo, Y-C. Chen, J-L. Liu, J-D. Leng, Z-S. Meng, P. Vrábel, M. Orendác and ML. Tong, Chem. Commun., 2012, 48, 12219-12221; Y-C. Chen, F-S. Guo, Y-Z. Zheng, J-L. Liu, J-D. Leng, R. Tarasenko, M. Orendác, J. Proleska, V. Sechovsky and M-L. Tong, Chem. Eur. J., 2013, 19, 13504-13510

10 G. Lorusso, M. Palacios, G. S. Nichol, E. K. Brechin, O. Roubeau and M. Evangelisti, Chem. Commun., 2012, 48, 7592-7594

11 G. Lorusso, J. W. Sharples, E. Palacios, O. Roubeau, E. K. Brechin, R. Sessoli, A. Rossin, E. K. Brechin, F. Tuna, E. J. L. McInnes, D. Collison and M. Evangelisti, Adv. Mater., 2013, 25, 4653-4656

12 Only limited increase of the magnetic over non-magnetic weight ratio can be expected with respect to the very dense framework material based on formate ions reported in ref. 11. On the other hand, and on basis of the magneto-structural considerations described herein, $\mathrm{Gd} \cdots \mathrm{Gd}$ ferromagnetic interactions significantly higher than that in Gd-ac (Ref. 7) are quite unlikely to be found, at least in carboxylatobridged systems.

13 G. Lorusso M. Jenkins, P. González-Monje, A. Arauzo, J. Sesé, D. Ruiz-Molina, O. Roubeau and M. Evangelisti, Adv. Mater., 2013, 25, 2984-2988

14 CrysalisPro, Agilent Technologies Inc.

15 SAINT, SADABS and SHELXTL, Bruker AXS Inc., Madison, Wisconsin, USA.

16 G. M. Sheldrick, Acta Cryst., 2008, A64, 112-122

17 F-S. Guo, J-D. Leng, J-L. Liu, Z-S. Meng and M-L. Tong, Inorg. Chem., 2012, 51, 405-413

18 CSD version 5.33; November $2011+4$ updates (August 2012)

19 L. Cañadillas-Delgado, O. Fabelo, J. Pasán, F. S. Delgado, F. Lloret, M. Julve and C. Ruiz-Pérez, Dalton Trans., 2010, 39, 7286-7293

20 M. E. Fisher, Am. J. Phys., 1964, 32, 343-346

21 the fit for compound 1 provides a $\chi^{2}$ of 0.009 , while for 2 the best fit gives a $\chi^{2}$ of 0.06

22 L. Cañadillas-Delgado, J. Pasán, O. Fabelo, M. Julve, F. Lloret and C. Ruiz-Perez, Polyhedron, 2013, 52, 321-332.

23 R. Baggio, R. Calvo, M. T. Garland, O. Peña, M. Perec and A. Rizzi, Inorg. Chem., 2005, 44, 8979-8987

24 a) S. T. Hatscher and W. Urland, Angew. Chem. Int. Ed., 2003, 42, 2862-2864; b) M. C. Favas, D. L. Kepert, B. W. Skelton and A. H. White, J. Chem. Soc. Dalton Trans., 1980, 454-458; c) A. Rohde and W. Urland, J. Alloys Compd., 2006, 408-412, 618-621; d) M. Hernández-Molina, C. Ruiz-Pérez, T. López, F. Lloret and M. Julve, Inorg. Chem., 2003, 42, 5456-5458; e) A. Rohde and W. Urland, Z. Anorg. Allg. Chem., 2005, 631, 417-420; f) L. Cañadillas-Delgado, O. Fabelo, J. Cano, J. Pasán, F. S. Delgado, F. Lloret, M. Julve and C. Ruiz-Pérez, CrystEngComm, 2009, 11, 2131-2142; g) N. Xu, W.
Shi, D-Z. Liao, S-P. Yan and P. Cheng, Inorg. Chem., 2008, 47, 8748-8756

25 a) J-P. Costes, J. M. Clemente-Juan, F. Dahan, F. Nicodème and M. Verelst, Angew. Chem. Int. Ed., 2002, 41, 323-325; b) D. John and W. Urland, Eur. J. Inorg. Chem., 2006, 3503-3509; c) D. John and W. Urland, Z. Anorg. Allg. Chem., 2005, 631, 2635-2637; d) D. John and W. Urland, Eur. J. Inorg. Chem., 2005, 4486-4489; e) A. Panagiotopoulos, T. F. Zafiropoulos, S. P. Perlepes, E. Bakalbassis, I. Masson-Ramade, O. Kahn, A. Terzis and C. P. Raptopoulou, Inorg. Chem., 1995, 34, 4918-4920; f) A. W-H. Lam, W-T. Wong, S. Gao, G. Wen and X-X. Zhang, Eur. J. Inorg. Chem., 2003, 149-163 